\title{
ESTIMATIVA DA TENDÊNCIA DIFERENCIAL DO CÓDIGO NOS RECEPTORES GNSS
}

Estimative of the differential code bias in the GNSS receivers

\author{
FABRICIO DOS SANTOS PROL \\ PAULO DE OLIVEIRA CAMARGO
}

\author{
Universidade Estadual Paulista Júlio de Mesquita Filho - UNESP \\ Programa de Pós-Graduação em Ciências Cartográficas \\ Departamento de Cartografia \\ Rua Roberto Simonsen, 305, Caixa Postal 468 \\ CEP: 19060-900 - Presidente Prudente - SP - Brasil \\ fabricioprol@hotmail.com; paulo@fct.unesp.br
}

\begin{abstract}
RESUMO
A tendência diferencial devido ao atraso do código (DCB) dos satélites e receptores é um dos parâmetros estimados, juntamente com o conteúdo total de elétrons (TEC), nos modelos ionosféricos que utilizam dados do GNSS. O IGS disponibiliza arquivos em formato IONEX que fornecem os valores dos DCB's dos satélites e receptores utilizados na modelagem do TEC a partir de uma rede GNSS global. Visando calcular o DCB para qualquer estação receptora, pertencente ou não a rede utilizada pelos centros de análise do IGS, este trabalho apresenta uma técnica de estimativa do DCB do receptor baseada em arquivos IONEX. O método estima o DCB do receptor a partir da média aritmética da combinação linear livre da geometria. O processamento é realizado com menos esforço computacional ao utilizado na modelagem ionosférica, pois não é necessário realizar a modelagem do TEC. Os DCB's estimados apresentaram concordância de 0,47 ns com os DCB's disponibilizados nos arquivos IONEX do CODE em estações pertencentes à região brasileira.
\end{abstract}

Palavras-chave: DCB; TEC; IONEX.

ABSTRACT
The differential code bias (DCB) is one estimated parameter in ionospheric models
using data from GNSS. DCB's values from satellites or receivers can be obtained 
from IONEX format files provided by IGS and these parameters are estimated using a global GNSS network. In this context, this paper presents an estimation technique of DCB based on the IONEX files, in order to compute a DCB for any local station. The method consists in applying the arithmetic mean of the linear combination of geometry free. The processing is performed with less computational effort than that used in the ionospheric modeling, because it is not necessary to use the TEC modeling. The DCB's were estimated with stations of Brazilian region and showed agreement of $0.47 \mathrm{~ns}$ in comparison to the DCB's provided by CODE.

Keywords: DCB; TEC; IONEX.

\section{INTRODUÇÃO}

O atraso no sinal GNSS (Global Navigation Satellite Systems) que ocorre em função da tendência diferencial do código (DCB - Differential Code Bias) é considerado um efeito instrumental decorrente da diferença entre o tempo de propagação das diferentes portadoras no hardware dos equipamentos. Atrasos adicionais ao DCB ocorrem devido à sincronização imperfeita na modulação do código na geração do sinal (satélite) e na demodulação do código (receptor) (HEGARTY et al., 2005; HONG, 2007).

Os DCB's do satélite e do receptor são dependentes da frequência do sinal e, portanto, não são eliminados nas combinações lineares entre as portadoras. Uma vez que o atraso ionosférico também depende da frequência do sinal, os DCB's podem ser considerados fontes de erros na estimativa do atraso ionosférico e nas quantidades de TEC (Total Eletron Content - Conteúdo Total de Elétrons).

A partir de medidas GNSS obtidas por uma rede de receptores de dupla frequência, diversos modelos foram desenvolvidos para a estimativa do TEC em conjunto com os DCB's. No Brasil, Aguiar e Camargo (2012), Sejas et al. (2010), Camargo (2009) e Veronez et al. (2007), desenvolveram modelos regionais baseados na estimativa do atraso ionosférico projetado na vertical de Pontos Ionosféricos (IPP - Ionospheric Pierce Point) localizados em uma camada fina de altitude constante. Proporcionando modelos capazes de estimar valores verticais de TEC (VTEC - Vertical TEC) e produzir arquivos em formato IONEX (IONosphere Map EXchange Format).

Com dados GNSS advindos de uma rede global, o IGS (International GNSS Service) deu início à produção de arquivos IONEX em 1998, que além das estimativas dos valores do VTEC, também inclui os DCB's dos satélites e de receptores da rede. Os VTEC dos arquivos IONEX dos centros de análises do IGS (CODE - Centre for Orbit Determination in Europe, JPL - Jet Propulsory Laboratory, UPC - Universitat Politècnica de Catalunya, ESA - European Space Agency) são apresentados em uma grade de resolução espacial de $5^{\circ} \times 2,5^{\circ} \mathrm{em}$ longitude e latitude, respectivamente, com uma resolução temporal de 2 horas.

A partir da grade de VTEC do IONEX, é possível estudar alguns fenômenos da ionosfera, assim como as investigações feitas por Matsuoka et al. (2013), 
referentes ao impacto de explosões solares na ionosfera. No dia da tempestade analisada, verificou-se um aumento no VTEC após o pôr do Sol em regiões ao Sul do Brasil.

Ademais, os arquivos IONEX podem ser utilizados na melhoria do posicionamento GNSS, como é mostrado nos testes realizados por Armendaris et al. (2009), que aplica as correções dos erros ionosféricos, nas observáveis, a partir dos mapas globais de TEC do IGS produzidos pelo CODE. Os resultados apresentaram uma melhora média no posicionamento por ponto, com relação ao processamento sem correção do efeito da ionosfera, da ordem de $77 \%$ na componente altimétrica e de $44 \%$ na planimétrica, sendo que foram utilizadas somente as observáveis da portadora $\mathrm{L}_{1}$.

Considerando a importância da estimativa dos DCB's em aplicações do GNSS, tanto na análise de fenômenos ionosféricos, bem como na influência da estimativa do TEC e, consequentemente, no posicionamento com o GNSS, este artigo descreve os princípios de alguns métodos de estimativa do DCB e apresenta a qualidade da técnica proposta para a estimativa do DCB do receptor, pertencente ou não, à rede IGS, que é utilizada pelos centros de análises na produção de arquivos ionosféricos em formato IONEX.

\section{ESTIMATIVA DA TENDÊNCIA DIFERENCIAL DO CÓDIGO}

Os satélites GNSS transmitem os sinais com, no mínimo, duas frequências, quase que simultaneamente. A diferença no tempo de transmissão do sinal GNSS pelas diferentes portadoras resulta em uma tendência no satélite. Um fenômeno similar ocorre no conjunto antenas/cabos/receptores, pois as portadoras percorrem os componentes no hardware dos equipamentos com velocidades distintas e então, o código é demodulado. Ao realizar a combinação entre as portadoras, torna-se acessível o atraso devido à tendência diferencial do código e, no caso das portadoras $\mathrm{L}_{1}$ e $\mathrm{L}_{2}$, o DCB $\left(\Delta b_{P}\right)$, em segundos, pode ser expresso por (HONG, 2007):

$$
\begin{gathered}
\Delta b_{P}^{S}=b_{P_{2}}^{S}-b_{P_{1},}^{S}, \\
\Delta b_{r, P}=b_{r, P_{2}}-b_{r, P_{1}},
\end{gathered}
$$

em que $b$ representa o tempo de propagação no hardware dos equipamentos, $s$ é o índice referente ao satélite, $r$ ao receptor, e $P$ se refere às observações de código da pseudodistância.

Uma vez que o DCB é decorrente da diferença entre o tempo de propagação das diferentes portadoras no hardware dos equipamentos, estes não são eliminados na combinação linear livre da geometria. Com isso, tal combinação pode ser empregada para estimar o DCB entre as portadoras (CAMARGO, 2009; CIRAOLO et al., 2007). A equação de observação da combinação linear livre da geometria para as portadoras $\mathrm{L}_{1}$ e $\mathrm{L}_{2}$ e medidas de código é dada por: 


$$
\left(P_{2}-P_{1}\right)=\left(I_{P 2}-I_{P 1}\right)+c\left(\Delta b_{P}^{S}+\Delta b_{r, P}\right)+\varepsilon_{P}
$$

O atraso ionosférico é representado por $I$, a velocidade da luz no vácuo é identificada por $c$ e os efeitos sistemáticos não modelados e aleatórios são apresentados por $\varepsilon$. Baseado na Equação (3) é possível realizar a estimativa do DCB em conjunto com um modelo de ionosfera.

Georgiadiou (1994) apresenta um método para estimar o DCB junto a um modelo ionosférico com apenas um receptor, em que os DCB's do satélite e do receptor são calculados em conjunto $\left(\Delta b_{P}^{S}+\Delta b_{r, P}\right)$. Com uso de uma rede de receptores, é possível determinar o DCB de cada um dos satélites e receptores; porém, o modelo matemático possui deficiência de característica. Camargo (2009) apresenta o modelo identificado Mod_Ion, no qual introduz a injunção sobre o conhecimento do DCB com respeito a um dos receptores da rede ou um dos satélites. Já os centros de análises do IGS introduzem a condição de que a média das estimativas dos DCB's dos satélites seja nula (zero-mean).

A partir dos arquivos IONEX, pode-se interpolar valores de VTEC para a região de abrangência da grade e estimar o atraso ionosférico no sinal GNSS. Substituindo o atraso ionosférico e o DCB do satélite (do arquivo IONEX) na Equação (3), pode-se estimar o DCB do receptor. Arikan et al. (2008) utiliza de tal recurso no método identificado IONOLAB-BIAS, na estimativa do DCB do receptor a partir de arquivos IONEX. Montenbruck et al. (2014) também utiliza arquivos IONEX do IGS e apresenta uma técnica para calcular o DCB do receptor baseada na média aritmética da combinação linear livre da geometria entre as medidas de código das portadoras.

Além desses métodos, pode-se considerar valores a priori do atraso ionosférico em épocas de mínima atividade solar sobre a região de interesse e, assim, realizar uma estimativa aproximada do DCB calculado em conjunto para o satélite e o receptor. Cornely (2013) menciona o método Zero TEC, em que o atraso ionosférico é considerado aproximadamente zero em períodos noturnos e o DCB conjunto (satélite e receptor) é calculado por meio da combinação linear livre da geometria, apresentada na Equação (3).

Uma alternativa para o cálculo do DCB, independente do modelo ionosférico, é realizar a Simples Diferença (SD) entre dois receptores dispostos em linhas de base curtas (CAMARGO et al., 2012). No entanto, o DCB será um valor relativo entre as estações e o impacto da ionosfera é considerado igual para os sinais GNSS rastreados simultaneamente em cada um dos receptores.

Destaca-se que a quantidade de TEC presente nos sinais GNSS é maior à medida que a distância entre o satélite e o receptor aumenta. Baseado nisso, Hong et al. (2008) elaborou um método em que as SD's são formadas entre receptores que estabelecem uma linha de base de até $200 \mathrm{~km}$. O método foi aplicado em estações da rede CORS (Continuously Operating Reference Stations) em Ohio e a 
discrepância média entre os valores estimados e os de referência foi de $4 \mathrm{~cm}$. Apesar de o método proporcionar valores próximos aos DCB's de referência, a SD estimada é suscetível a efeitos devido, principalmente, ao multicaminho e ruídos.

A observável da SD livre dos efeitos da ionosfera, bem como livre da geometria e de efeitos da propagação do sinal, pode ser obtida a partir de linhas de base zeradas (zero baselines) com uso de um divisor de sinais GNSS. Nesse caso, uma única antena é conectada a diferentes receptores e a SD retém apenas o erro do relógio dos receptores e os termos de tendência (MONTENBRUCK, 2011). AlShaery et al. (2013) utiliza tal recurso para estimar as tendências dos receptores, por meio de medidas de fase, e fixa as ambiguidades com cerca de $92 \%$ de sucesso no processamento GNSS combinando observáveis GPS (Global Positioning System) e GLONASS (Globalnaya Navigatsionnaya Sputnikovaya Sistema).

Outra abordagem para a estimativa do DCB é a partir da calibração do receptor. No entanto, o DCB medido é suscetível a ruídos, assim como mostrado por Banville et al. (2008), que realiza a calibração das tendências de fase a partir de um simulador de sinais e considera que os DCB's são propagados em demais parâmetros no processo de ajustamento, tal como em uma parte intrínseca ao erro do relógio dos receptores e nas coordenadas da estação.

\subsection{Estimativa dos DCB's com Modelos Ionosféricos}

Em razão da propriedade dispersiva da ionosfera aos sinais GNSS transmitidos em, no mínimo, duas frequências distintas, é possível elaborar modelos que descrevem o atraso ionosférico. $\mathrm{O}$ atraso ionosférico ocorre principalmente devido à densidade de elétrons livres $\left(n_{e}\right)$ presente no trajeto do sinal GNSS. Como na prática é difícil de obter a densidade de elétrons, grande parte dos modelos estimam a integral da densidade de elétrons ao longo do trajeto entre o satélite $(s)$ e a antena receptora $(r)$, ou seja, estimam o TEC em coluna cuja área da seção transversal equivale a $1 \mathrm{~m}^{2}$ (LEICK, 1995; SEEBER, 2003).

$$
T E C=\int_{r}^{s} n_{e} d s
$$

A relação entre a densidade de elétrons e a refração ionosférica é apresentada por Seeber (2003), em que a refração da ionosfera é proporcional ao TEC e inversamente proporcional ao quadrado da frequência $(f)$ do sinal GNSS na portadora $(i)$ :

$$
I_{P, i}=\frac{40,3}{f_{i}^{2}} T E C
$$




$$
I_{\varphi, i}=-\frac{40,3}{f_{i}^{2}} T E C
$$

A refração ionosférica relacionada com o código (Equação 5) é usualmente denominada de atraso de grupo, que junto ao avanço da fase da onda portadora $(\varphi)$ (Equação 6) são um dos principais efeitos sistemáticos sobre o posicionamento GNSS. Devido a aproximações no índice de refração para a obtenção das Equações (5) e (6), descritas por Hartree-Appleton (LEICK, 1995), o atraso ionosférico, utilizado em grande parte dos modelos ionosféricos, representa somente os efeitos de $1^{a}$ ordem da ionosfera. Os efeitos das demais ordens são comumente negligenciados, pois a dimensão dos erros de $2^{\mathrm{a}}$ ordem é centimétrica e de $3^{\mathrm{a}}$ ordem, milimétrica (MARQUES et al., 2011).

A partir da combinação linear livre da geometria (Equação 3), e relacionando o atraso ionosférico de código com o TEC (Equação 5), pode-se obter uma relação entre as observáveis GNSS e o TEC. No caso das medidas de código nas portadoras $\mathrm{L}_{1}$ e $\mathrm{L}_{2}$, tem-se (CIRAOLO et al., 2007):

$$
F\left(P_{2}-P_{1}\right)=T E C+F\left[c \Delta b_{r, P}+\mathbf{c} \Delta b_{P}^{s}+\varepsilon_{P}\right],
$$

com:

$$
F=\frac{f_{1}^{2} f_{2}^{2}}{40,3\left(f_{1}^{2}-f_{2}^{2}\right)}
$$

Nos modelos ionosféricos, habitualmente, faz-se a simplificação de que o TEC presente nos sinais GNSS está contido em uma fina camada de altitude $h_{m}$, sendo projetado no ponto ionosférico (IPP - Ionospheric Pierce Point), ponto este formado pela intersecção do vetor receptor/satélite com uma camada infinitesimal da ionosfera que se localiza, em geral, entre 350 e $450 \mathrm{~km}$ acima da superfície terrestre. Para a consideração da camada fina, os modelos ionosféricos estimam o TEC na direção vertical (VTEC) dos IPP's a partir de uma função de mapeamento. Na função de mapeamento geométrica padrão, tem-se (HOFMANN-WELLENHOF; COLLINS, 2001):

sendo:

$$
V T E C=\cos \left(z^{\prime}\right) T E C,
$$

$$
\operatorname{sen}\left(z^{\prime}\right)=\frac{r_{m}}{r_{m}+h_{m}} \operatorname{sen}(z)
$$

Em que $z$ é o ângulo zenital do trajeto entre o satélite e o receptor, $z^{\prime}$ é o ângulo zenital entre o satélite e o IPP, $r_{m}$ é o raio médio da Terra e $h_{m}$ representa a altura média da camada da ionosfera. A Figura 1 ilustra a geometria utilizada nos 
modelos ionosféricos de fina camada bem como os parâmetros envolvidos na projeção do IPP.

Figura 1 - Geometria do modelo ionosférico de fina camada.

Fonte: Adaptado de Hofmann-Wellenhof et al (2001).

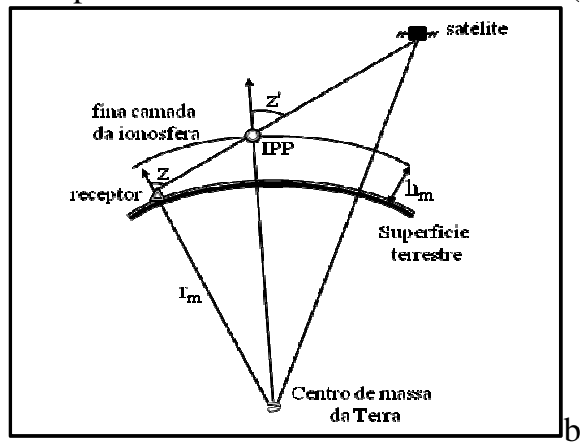

Ao substituir a Equação (9) em (7), obtém-se a principal equação de observação para modelagem da ionosfera com o GNSS:

$$
F\left(P_{2}-P_{1}\right)=\frac{V T E C}{\cos \left(z^{\prime}\right)}+F\left[c \Delta b_{r . P}+c \Delta b_{P}^{S}+\Delta \varepsilon_{P}\right]
$$

O DCB é estimado no processo de estimação dos parâmetros e devido à natureza periódica do efeito da refração ionosférica, o VTEC é, normalmente, modelado por funções matemáticas de superfície, tais como séries, polinômios e harmônicos esféricos. Destaca-se que em vez da pseudodistância original, pode-se utilizar a pseudodistância filtrada pela fase da portadora (CAMARGO, 2009), fazendo-se necessário um algoritmo de detecção de perda de ciclo (HOFMANNWELLENHOF et al, 2001).

\subsection{Estimativa do DCB do Receptor Baseada na Média Aritmética}

Uma abordagem alternativa à modelagem ionosférica para estimar o DCB de um receptor pode ser realizada a partir do TEC e dos DCB's dos satélites que constam em arquivos IONEX. Com base na combinação linear livre da geometria e dos arquivos IONEX, o princípio do método é apresentado na seguinte equação:

$$
c \Delta b_{r, P}=\frac{1}{n} \sum_{k=1}^{n}\left[\left(P_{2}-P_{1}\right)_{k}-\frac{T E C_{k}}{F}-c \Delta b_{P}^{S}\right]
$$

A Equação (12) representa a média aritmética dos valores calculados para cada um dos $(k)$ instantes medidos, em que representa o número de observações. Após 
calcular a posição do IPP do respectivo sinal GNSS e interpolar os valores de VTEC do IONEX, aplica-se a função de mapeamento para converter o VTEC em $T E C_{k}$. Os valores dos DCB's dos satélites $\left(\Delta b^{s}\right)$ são obtidos do arquivo IONEX.

A média aritmética da combinação linear livre da geometria para um período de, aproximadamente, 24 horas de observação, proporciona a estimativa do DCB do receptor com base em arquivos IONEX. O processamento é realizado com menos esforço computacional ao utilizado na modelagem ionosférica, pois, não será necessário realizar a modelagem do TEC. Destaca-se que o baixo nível de ruído nas medidas de código é essencial na estimativa do DCB do receptor, o que pode ser obtido com o processo de filtragem do código pela fase (JIN, 1996).

\section{EXPERIMENTOS, RESULTADOS E ANÁLISES}

Nos experimentos realizados, foram utilizados arquivos RINEX (Receiver Independent Exchange Format) de janeiro a outubro de 2013, das seguintes estações da Rede Brasileira de Monitoramento Contínuo dos Sistemas GNSS (RBMC): BOAV, BOMJ, BRAZ, BRFT, CEFE, CHPI, ILHA, POVE, SALU, SAVO e UFPR. As estações selecionadas são localizadas em diversas regiões latitudinais da ionosfera e compreendem todas as estações brasileiras utilizadas pelo CODE na modelagem da ionosfera para os dias de interesse. Destaca-se que a qualidade do DCB estimado é altamente dependente da qualidade do mapa ionosférico. Desta forma, os mapas do CODE foram utilizados, pois foram produzidos com maior quantidade de estações em região brasileira, em comparação com as outras agências do IGS.

A fim de estimar DCB's de receptores não utilizados na produção do IONEX do CODE, as estações da RBMC: TOPL, MSCG e SMAR selecionadas por se situarem em regiões geográficas com diferentes condições ionosféricas. No que se refere às variações latitudinais, TOPL se localiza próximo ao equador geomagnético, MSCG é instalada sobre região da crista da anomalia equatorial, e SMAR pode ser considerada uma estação em região de baixa atividade ionosférica.

Aplicou-se máscara de elevação igual a $60^{\circ}$ a fim de minimizar efeitos de multicaminho (ARIKAN et al., 2008) e, principalmente, erros devido à projeção dos IPP's. Os valores de VTEC dos arquivos IONEX foram interpolados temporalmente e espacialmente para a posição dos IPP's, a partir da interpolação bilinear e obtevese um valor de VTEC para cada instante medido. Em cada instante, foi feito o cálculo da combinação linear apresentada na Equação (12), incluindo os valores de TEC e DCB dos satélites do GPS. Ao final da leitura do arquivo RINEX, calculouse a média aritmética das combinações lineares entre as medidas de código filtrado pela fase da onda portadora e obteve-se o DCB do receptor no respectivo dia.

$\mathrm{Na}$ etapa de pré-processamento foi realizado o método de Posicionamento por Ponto Simples (PPS) a fim de estimar a posição da estação, o azimute e elevação do satélite e a respectiva posição do IPP para a altitude informada no IONEX, possibilitando converter os valores de VTEC para TEC com a função de mapeamento geométrica padrão. Além disso, a pseudodistância foi filtrada pela fase 
Prol. F. S. ; Camargo, P. O.

com o método proposto em Jin (1996) e a detecção de perdas de ciclo foi realizada com o método baseado na ambiguidade da widelane (BLEWITT, 1990).

As Figuras 2 a 12 apresentam a comparação dos valores dos DCB's calculados pelo CODE e os valores estimados na técnica da média aritmética, de forma a quantificar a qualidade do método. Em alguns casos, as estações selecionadas não foram utilizadas na produção dos arquivos IONEX, ou então, há falta de dados nos arquivos RINEX.

Figura 2 - DCB e diferença em BOAV. Figura 3-DCB e diferença em CEFE.
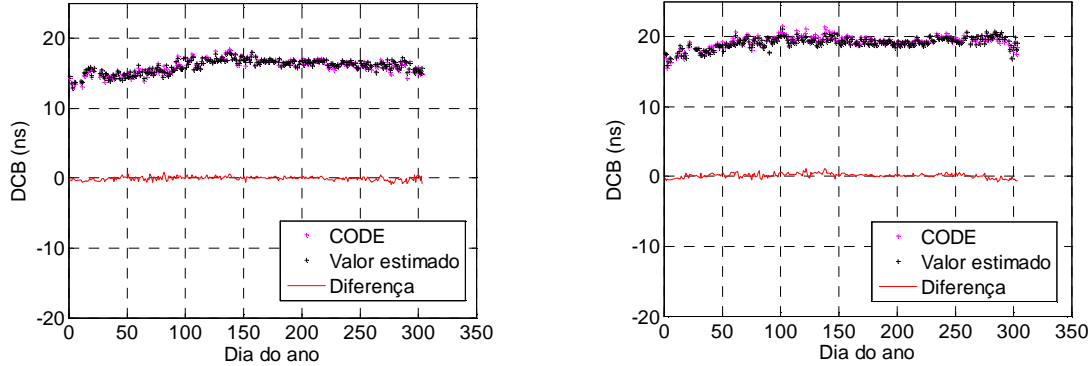

Figura 4 - DCB e diferença em BOMJ. Figura 5 - DCB e diferença em CHPI.
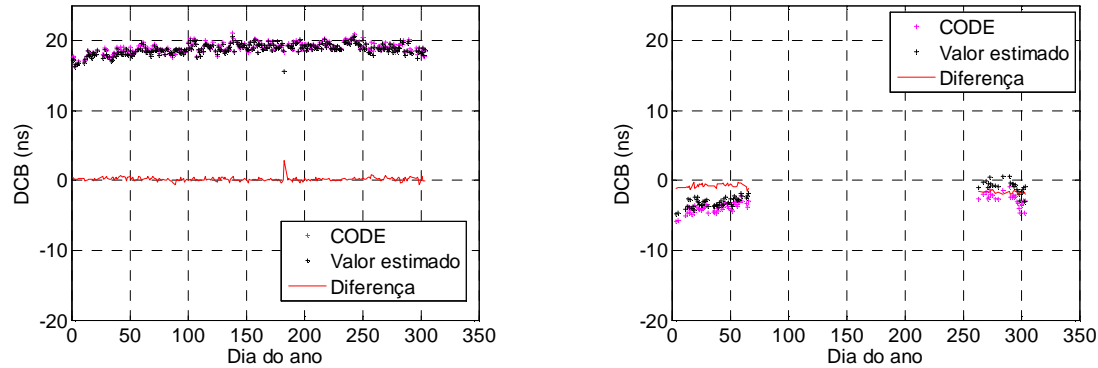

Figura 6 - DCB e diferença em BRFT.

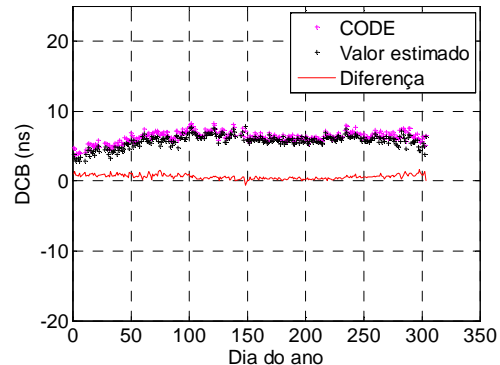

Figura 7 - DCB e diferença em ILHA.

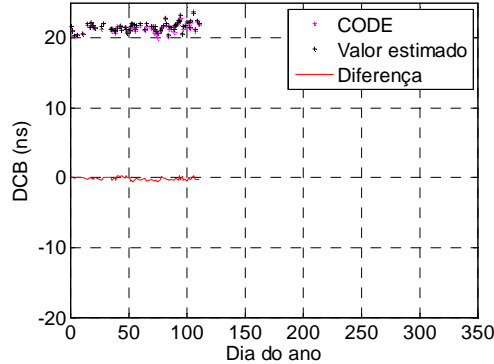

Bol. Ciênc. Geod. sec. Artigos, Curitiba, v. 20, no 4, p.735-749, out-dez, 2014. 
Figura 8 - DCB e diferença em POVE.

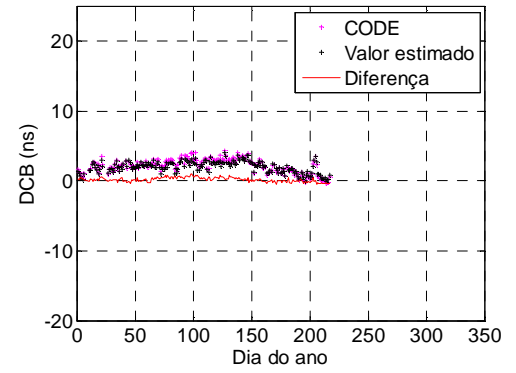

Figura 10 - DCB e diferença em SALU.

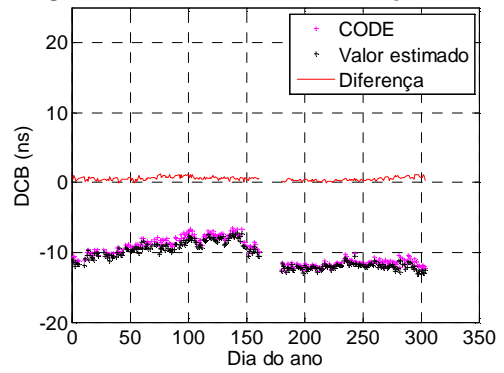

Figura 9 - DCB e diferença em SAVO.

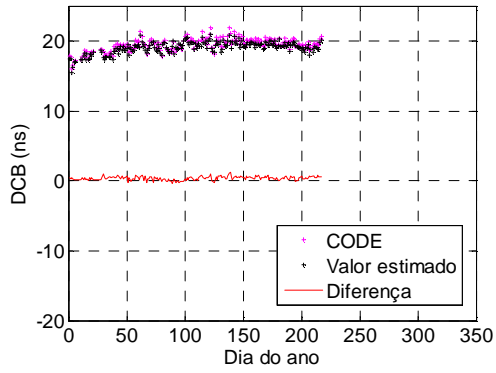

Figura 11 - DCB e diferença em UFPR.

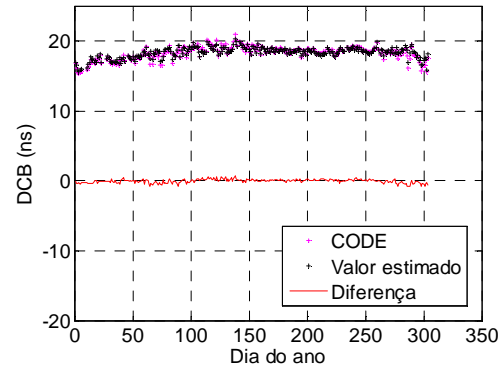

Figura 12 - DCB e diferença em BRAZ.

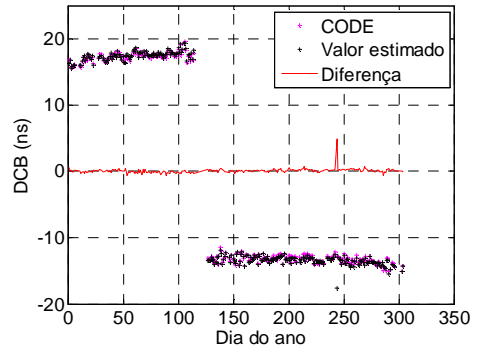

Observa-se, nas Figuras 2 a 12, que a diferença entre os DCB's (CODE - valor estimado) tendem a zero na maioria dos dias. Além disso, o método proposto acompanha as variações dos DCB's para todas as estações, inclusive nas estações BRFT, CHPI e POVE, as quais apresentam maior dispersão diária dos DCB's. Na estação CHPI (Figura 5), nota-se que após o dia 263 de 2013, apresentou maiores discrepâncias em relação aos dias anteriores, o que ocorreu devido à mudança do conjunto de equipamentos da fabricante Ashtech pelo receptor JAVAD TRE-G3TH DELTA e antena TPSCR-G3, no dia 30/07/2013 (dia 211). Na estação BRAZ 
(Figura 12), o salto no gráfico é referente à troca do receptor TRIMBLE NETRS por um TRIMBLE NETR8, no dia 25/04/2013.

A partir do conjunto de DCB's diários, calculou-se o desvio padrão em cada mês a fim de quantificar a dispersão dos DCB's estimados com o método apresentado e os disponibilizados pelo CODE (Figura 13).

Figura 13 - Desvio padrão mensal dos DCB's.

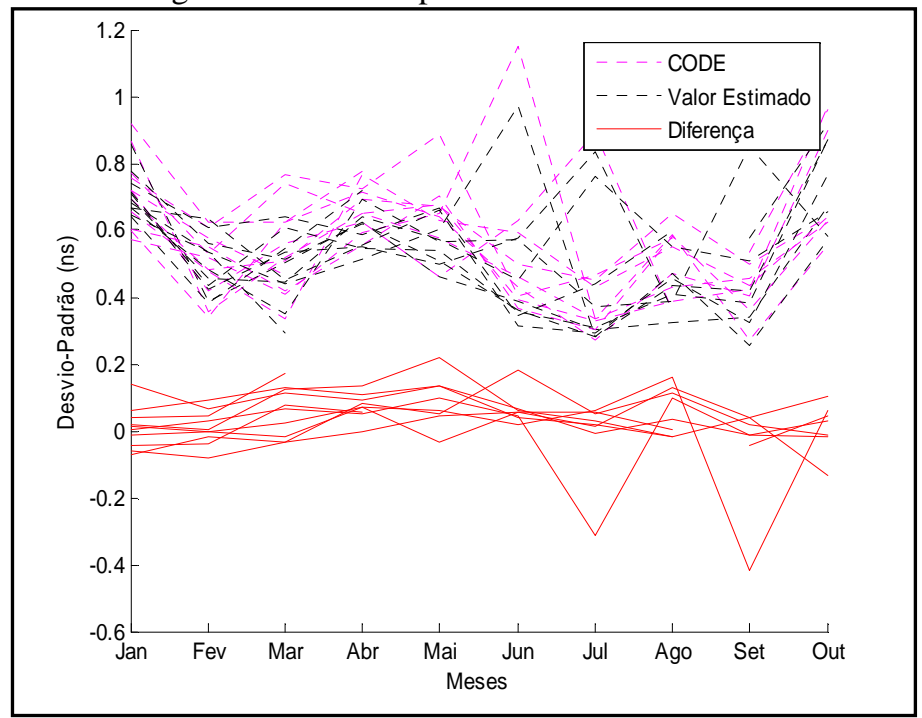

Os desvios padrão mensais dos DCB's estimados pelo CODE e os obtidos pela média aritmética variam entre $\pm 0,2$ ns e $\pm 1,2$ ns (Figura 13), onde as linhas tracejadas representam os desvios padrão das estações ao longo dos meses e a diferença dos desvios padrão mensais é apresentada pela linha contínua. A diferença da dispersão dos resultados tende a zero, e o desvio padrão é da ordem de $\pm 0,2 \mathrm{~ns}$.

A Tabela 1 apresenta as estatísticas das diferenças dos DCB's, ou seja, o erro médio quadrático (RMSE - Root Mean Square Error), valores máximos (Max) e mínimos (Min) das diferenças dos DCB's e desvio padrão médio $\left(\sigma_{\text {médio }}\right)$ das dispersões mensais dos DCB's estimados com a média aritmética.

Apesar de algumas discrepâncias atingirem um valor máximo de 4,78 ns (Tabela 1), os experimentos mostram que o método apresenta um erro médio quadrado da ordem de $0,47 \mathrm{~ns}$, com uma dispersão média mensal de $\pm 0,54 \mathrm{~ns}$. A precisão obtida em nanosegundos representa 1,5 TECU (TEC Units), compatível com a precisão com que os valores de TEC são estimados pelo IGS, que é de 2 à 8 TECU (IGSCB, 2013). Em que 1 TECU induz a um erro de cerca de 0,16 m, 0,27 m e $0,28 \mathrm{~m}$ nas observáveis das portadoras $\mathrm{L}_{1}, \mathrm{~L}_{2}$ e $\mathrm{L}_{5}$, respectivamente. 
Tabela 1 - Estatísticas das diferenças dos DCB's e desvio padrão médio.

\begin{tabular}{l|c|c|c|c}
\hline Estação & RMSE $(\mathbf{n s})$ & Max $(\mathbf{n s})$ & Min $(\mathbf{n s})$ & $\boldsymbol{\sigma}_{\text {médio }}(\mathbf{n s})$ \\
\hline BOAV & 0,28 & 0,78 & $-0,85$ & 0,48 \\
\hline BOMJ & 0,32 & 2,87 & $-0,69$ & 0,57 \\
\hline BRAZ & 0,37 & 4,78 & $-0,65$ & 0,55 \\
\hline BRFT & 0,67 & 1,60 & $-0,61$ & 0,51 \\
\hline CEFE & 0,33 & 1,06 & $-0,72$ & 0,53 \\
\hline CHPI & 1,22 & 0,31 & $-2,05$ & 0,60 \\
\hline ILHA & 0,28 & 0,30 & $-0,69$ & 0,57 \\
\hline POVE & 0,36 & 1,00 & $-0,49$ & 0,56 \\
\hline SALU & 0,59 & 1,25 & $-0,09$ & 0,53 \\
\hline SAVO & 0,45 & 1,28 & $-0,32$ & 0,58 \\
\hline UFPR & 0,28 & 0,73 & $-0,83$ & 0,52 \\
\hline MÉDIA & 0,47 & 1,45 & $-0,73$ & 0,54 \\
\hline
\end{tabular}

A Figura 14 apresenta a estimativa dos DCB's dos receptores das estações TOPL, MSCG e SMAR da RBMC. Na estimativa dos DCB's dessas estações, foi utilizado o método apresentado.

Figura 14 - DCB's estimados das estações TOPL, MSCG e SMAR da RBMC.

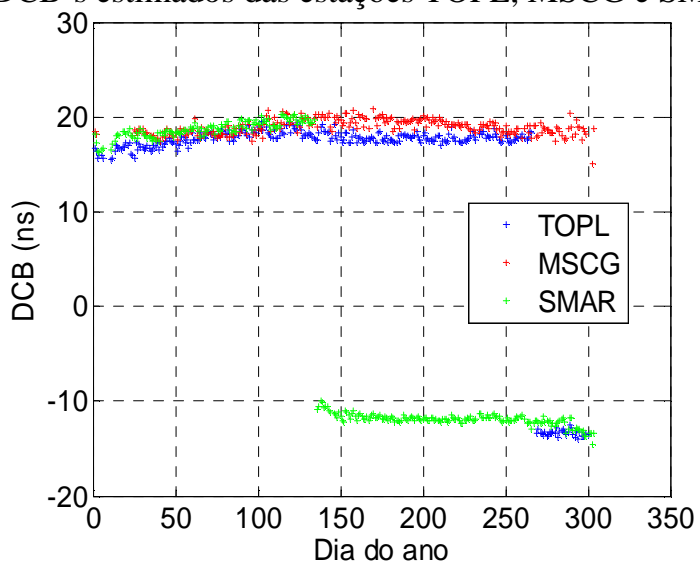

Os resultados apresentaram pouca dispersão para os DCB's estimados no período de dados em que não houve mudanças nos equipamentos, em que as médias mensais dos desvios padrão são de 0,42 ns (TOPL), 0,54 ns (MSCG) e 0,43 ns (SMAR). Verifica-se que o método detectou as mudanças dos receptores instalados em TOPL e SMAR, onde o salto no gráfico que representa o DCB da estação TOPL se refere à mudança do receptor TRIMBLE NETRS para o TRIMBLE NETR8 em 25/09/2013. Em SMAR, houve troca do receptor TRIMBLE NETRS para o TRIMBLE NETR8 em 15/05/2013. 


\section{CONCLUSÕES}

Os DCB's não são eliminados nas combinações lineares livres da geometria, o que influencia diretamente na determinação do atraso ionosférico. A partir de um processo de estimativa de parâmetros, os DCB's dos receptores e dos satélites podem ser estimados em conjunto com o TEC no modelo ionosférico, possibilitando a produção de arquivos IONEX. Visando estimar o DCB de receptores a partir de arquivos IONEX, um método baseado na média aritmética da combinação linear livre da geometria foi aplicado também em receptores que não fazem parte da modelagem para a produção do arquivo IONEX.

O método apresentado para a estimativa de DCB's de receptores foi avaliado a partir de arquivos IONEX disponibilizados pelo CODE e dados diários de arquivos RINEX da RBMC. A proposta mostrou-se condizente com os valores calculados pelo CODE ao nível de, aproximadamente, $0,47 \mathrm{~ns}$ com dispersão média de 0,54 ns, nos desvios padrão mensais (Janeiro a Outubro de 2013). Além disso, o método apresentou dispersão média de 0,46 ns nas estimativas de DCB's em estações não utilizadas na produção dos arquivos IONEX do CODE. Assim, ao aplicar a técnica da média aritmética e estimar valores dos DCB's dos receptores, é possível calcular o TEC diretamente.

Em trabalhos futuros pretende-se estimar as DCB's de todas as estações da RBMC, bem como realizar uma análise dos DCB's estimados a partir dos arquivos IONEX de todos os centros de análise do IGS: CODE, JPL, UPC e ESA; e também dos IONEX's produzidos a partir da combinação dos resultados proporcionados pelos centros de análise. Além disso, pretende-se aplicar o método apresentado para as diferentes constelações do GNSS.

\section{AGRADECIMENTOS}

Os autores agradecem a bolsa de Mestrado da CAPES e ao CNPq por auxílio à pesquisa e bolsa produtividade em pesquisa (Processos: 480103/2010-0 e 306719/2010-0, respectivamente), a FAPESP (Processo: 2006/04008-2) e ao IBGE pelo fornecimento dos dados GNSS e ao CODE por fornecer os arquivos IONEX.

\section{REFERÊNCIAS}

AGUIAR, C. R.; CAMARGO, P. O. Resolução espacial da grade ionosférica e do GIVE. Boletim de Ciências Gedésicas, Curitiba, v. 18, n. 3, p. 464-479, 2012. AL-SHAERY, A. ; ZHANG, S. ; RIZOS, C. An enhanced calibration method of GLONASS inter-channel bias for GNSS RTK. GPS Solutions, v. 17, p. 165173, 2013.

ARIKAN, H.; NAYIR, U. SEZEN; ARIKAN, O. Estimation of single station interfrequency receiver bias using GPS-TEC. Radio Science, v. 43, 2008. doi:10.1029/2007RS003785.

ARMENDARIS, O. C.; MATSUOKA, M. T.; CAMARGO, P. O. Desempenho do modelo global da ionosfera do IGS: Avaliação no posicionamento por ponto na 
região sul do Brasil em período de alta atividade solar. Boletim de Ciências Geodésicas, v.15, n.2, p. 208-223, 2009.

BANVILLE, S. ; SANTERRE, R. ; COCARD, M. ; LANGLEY, R. B. Satellite and receiver phase bias calibration for undifferenced ambiguity resolution. In: Proceedings of ION NTM, San Diego, California, 2008.

BLEWITT, G. An automated editing algorithm for GPS data. Geophys. Res. Lett., v. 17, n. 3, p. 199-202, 1990.

CAMARGO, P. O. Quality of TEC estimated with Mod_Ion Using GPS and GLONASS data. Mathematical Problems in Engineering, 2009, doi:10.1155/2009/794578.

CAMARGO, P. O.; ARANA, D. ; PEREIRA, V. A. S. Erro sistemárico relativo devido ao hardware dos receptores. In: III Simpósio Brasileiro de Geomática, 2012, Presidente Prudente. II Simpósio Brasileiro de Geomática e V Colóquio Brasileiro de Ciências Geodésicas, Presidente Prudente, v. 1, p. 268-273, 2012. CIRAOLO, L. ; AZPILICUETA, F. ; BRUNINI, C. ; MEZA, A. ; RADICELLA, S. M. Calibration errors on experimental slant total electron content (TEC) determined with GPS. Journal of Geodesy. v. 81, n. 2, p. 111-120, 2007.

CORNELY, P. R. Receiver Biases in Global Positioning Satellite Ranging. InTech, 2013. doi: $10.5772 / 55567$.

GEORGIADIOU, Y. Modelling the ionosphere for an active control network of GPS stations. LGR-Series - Publications of Delft Geodetic Computing Centre, Delft University of tecnology, n. 7, 1994.

HEGARTY, C. ; POWERS, E. ; FONVILLE, B. Accouting for Timing Biases Between GPS, Modenized GPS, and Galileo Signals. In: 36th Annual Precise Time and Time Interval (PTTI) Systems and Applications Meeting, Washington, D.C., p. 307-318, 2005.

HOFMANN-WELLENHOF, B. ; LICHTENEGGER, H. ; COLLINS, J.. Positioning System: theory and practice. 5. ed. New York: Springer-Verlag, 382 p., 2001.

HONG, C. Efficient GPS receiver DCB estimation for ionosphere modeling using satellite-receiver geometry changes. 2007. PhD Thesis - Ohio State University, Ohio.

HONG, C. ; GREJNER-BRZEZINSKA, D. A. ; KWON, J. H.. Efficient GPS receiver DCB estimation for ionosphere modeling using satellite-receiver geometry changes. Earth Planets Space E-letter, v. 60, p. 25-28, 2008.

IGSCB (International GPS Service Central Bureau) - online - endereço eletrônico: $<$ http://igscb.jpl.nasa.gov/components/prods.html>. Acesso em dezembro de 2013.

JIN, X. X. Theory of carrier adjusted DGPS positioning approach and some experimental results.1996. PhD Thesis - Delft University of Technology, Delft.

LEICK, A. GPS Satellite Surveying. 2. ed., New York: John Wiley \& Sons, 560 p., 1995. 
MARQUES, H. A. ; MONICO, J. F. G. ; AQUINO, M. RINEX HO: second- and third-order ionospheric corrections for RINEX observation files, GPS Solut., v.15, n. 3, p. 305-314, 2011.

MATSUOKA, M. T. ; COLLISCHONN, C. ; KLEIN, I. ; CAMARGO, P. O. ; PEREIRA, V. A. S. Impacto de tempestade geomagnética na Ionosfera e no posicionamento com GNSS: Estudo de caso para 20 de novembro de 2003 na Região brasileira. Boletim de Ciências Geodésicas, Curitiba, v. 19, p. 14-33, 2013.

MONTENBRUCK, O. ; HAUSCHILD, A. ; HESSELS, U. Characterization of GPS/GIOVE sensor stations in the CONGO network. GPS Solutions. v. 15, n. 3, p. 193-205, 2011.

MONTENBRUCK, O. ; HAUSCHILD, A. ; HESSELS, U. Differential Code Bias Estimation using Multi-GNSS Observations and Global Ionosphere Maps. In: Proceedings of ION ITM, p. 27-29, 2014.

SEEBER, G. Satellite Geodesy: Foundations, Methods and Aplications. Berlin, New York: Walter de Gruyter, 589 p., 2003.

SEJAS, M. I. ; KRUEGER, C. P. ; HECK, B. Avaliação de um modelo ionosférico local em redes GPS: estudo de caso para um período de baixa atividade solar. Boletim de Ciências Gedésicas, Curitiba, v. 16, n. 2, p. 253-276, 2010.

VERONEZ, M. R. ; SEGANTINE, P. C. L. ; THUM, A. B. Modelo ionosférico regional aplicado ao sistema de posicionamento global - GPS. Gaea, Unisinos, v. 3, p. 18-26, 2007.

(Recebido em março de 2014. Aceito em maio de 2014). 